\title{
Water quality and phytoplankton diversity of high altitude wetland, Dodi Tal of Garhwal Himalaya, India
}

\begin{abstract}
Water quality and phytoplankton diversity of high altitude (3,075 above m.a.s.1.) wetland Dodi Tal were monitored for a period of November 2015 to October 2016. A total of 47 species belonging to 43 genera of four families (Bacillariophyceae; Chlorophyceae; Cyanophyceae; Dinophyceae) of phytoplankton were encounted during the study. Bacillariophyceae was the dominant family representing 20 genera followed by Chlorophyceae (16 genera), Cyanophyceae (4 genera) and Dinophyceae (3 genera). A highly significant ( $\mathrm{F}=14.59$; $\mathrm{p}=1.43 \mathrm{E}^{-08}$ ) seasonal variation in the abundance of phytoplankton community of Dodi Tal was recorded. Maximum abundance of phytoplankton $\left(1,270 \pm 315.00 \mathrm{ind}^{\left.-1^{-1}\right)}\right.$ was found in autumn season and minimum (433.00 \pm 75.00 ind..$^{-1}$ ) in monsoon season. Multiple regression analysis made between density of phytoplankton and environmental variables revealed that the abundance of phytoplankton has a negative correlation with TDS, alkalinity, dissolved oxygen, $\mathrm{pH}$ and Chlorides. However, it has a positive correlation with water temperature phosphates and nitrates. Shannon Wiener diversity index was recorded maximum (4.09) in autumn season and minimum (3.59) in monsoon season. Canonical Correspondence Analysis (CCA) was also calculated between physico-chemical variables and phytoplankton diversity for assessing the effect of physico- chemical variables on various taxa of phytoplankton.
\end{abstract}

Keywords: high altitude wetland, physico-chemical characteristics, phytoplankton diversity, canonical correspondence analysis
Volume 2 Issue 6 - 2018

\author{
Ramesh C Sharma, Sushma Singh \\ Department of Environmental Sciences, Hemvati Nandan \\ Bahughuna Garhwal University, India
}

\begin{abstract}
Correspondence: Sushma Singh, Department of Environmental Sciences, Hemvati Nandan Bahughuna Garhwal University, (A Central University) Srinagar, Garhwal, 246174, Uttarakhand, India, Email susmasingh I66I@gmail.com
\end{abstract}

Received: July 31, 2018 | Published: November 05, 2018

\section{Introduction}

The Dodi Tal is one of the most beautiful high altitude wetlands of Garhwal Himalaya. It is the important destination of large number of trekkers, hikers, tourists and shepherds. It is an important source of drinking water for these people in addition to wild animals. Thus, the study on water quality and phytoplankton diversity is of paramount importance.

High altitude wetlands and their phytoplankton have been investigated since the early nineteenth century. Important contributions on the phytoplankton diversity of aquatic ecosystem of the world have been made by several workers. Tian et al. ${ }^{1}$ studied the variations of cyanobacteria with different environmental conditions in Nansi Lake, China. Fott et al. ${ }^{2}$ studied phytoplankton in three Tatra mountain lakes, Poland. Tolotti ${ }^{3}$ studied on phytoplankton and littoral diatoms communities in high mountain lake, Italy. Some of the studies on the phytoplankton of high altitude wetlands from the India have also been made. Ganai et al. ${ }^{4}$ studied the phytoplankton diversity of Wular Lake, Kashmir. Bhat et $a .^{5}$ conducted study on ecology and biodiversity of Pangong Lake. Laskar \& Gupta ${ }^{6}$ contributed on phytoplankton community and limnology of Chatla wetland, Assam. Several workers have studied the water quality and phytoplankton diversity. Jindal et al. ${ }^{7}$ worked on Prashar Lake, Himachal Pradesh. Jindal et al. ${ }^{8}$ studied three freshwater lakes of Mandi, Himachal Pradesh. Singh et al. ${ }^{9}$ and Singh et al. ${ }^{10}$ studied the high altitude lakes of Lahaul-Spiti of Himachal Pradesh. Negi \& Rajput ${ }^{11}$ studied the diversity of phytoplankton in relation to different environmental variables in Nainital lake of Kumaon Himalaya, Uttarakhand. Gupta et al. ${ }^{12}$ worked on water quality and its relation with phytoplanktonic species of a freshwater high altitude lake, Nainital, Uttarakhand. Sharma \& Tiwari $^{13}$ studied the physico-chemical variables of water and their relation with phytoplankton in Nachiketa Tal, Uttarakhand.
But no attempt has been made to study water quality and phytoplankton diversity of Dodi Tal of Garhwal Himalaya. Therefore, the present contribution on base line data of water quality and phytoplankton diversity can be used as reference for further monitoring and water quality of the Dodi Tal of Garhwal Himalaya.

\section{Material and methods}

Dodi Tal is is located at latitude $30^{\circ} 52^{\prime} 31.99$ ' N and longitude $78^{\circ} 31^{\prime} 12.47^{\prime \prime} \mathrm{E}$, in Uttarkashi of Uttarakhand state (Figure 1). It is surrounded by an extremely dense forest of Quercus spp L., Cedrus deodara (Roxb. ex D. Don) and Rhododendron spp L. Dodi Tal is a semi-triangular in shape spread within the circumference of around $1.5 \mathrm{~km}$. The approximate length of the wetland is $248 \mathrm{~m}$ and width $152 \mathrm{~m}$ at its broadest points with maximum depth of $19.97 \mathrm{~m}$ and average depth of $9.98 \mathrm{~m}$. The Brown Trout (Salmo trutta Fario; Family: Salmonidae) has been introduced in this high altitude wetland. The major source of water of Dodi Tal is the several natural springs in addition to one perennial stream. The only outlet of Dodi Tal forms the river Asi Ganga, an important tributary of Bhagirathi River that merges with the Bhagirathi at Gangori near Uttarkashi.

\section{Collection of samples and their analysis}

The water of the wetland was sampled for a period of November, 2014 to October, 2016 except the months of February and March. The high altitude wetland Dodi Tal was not accessible during these months due to heavy snowfall. Water samples were collected from the four different sampling sites $\left(\mathrm{S}_{1}, \mathrm{~S}_{2}, \mathrm{~S}_{3}\right.$ and $\left.\mathrm{S}_{4}\right)$ during 8:00 am to 10:00 am. The sampling site $\mathrm{S}_{1}$ is the inlet or the source of water in the wetland; $\mathrm{S}_{2}$ is the outlet; $\mathrm{S}_{3}$ is close to the Ganesh temple; and $\mathrm{S}_{4}$ is in proximately of dense forest. A total of nineteen physico-chemical parameters were measured at each sampling site for assessing the water quality of the wetland. Water temperature was recorded with the help of the Digital 
Thermometer $\left(-50^{\circ} \mathrm{C}\right.$ to $\left.+300^{\circ} \mathrm{C}\right)$. Electrical conductivity and $\mathrm{pH}$ of the water were measured with the help of the Toshcon Multiparameter Analyser (Model No. TPC-17). Nitrates, phosphates and sulphates were determined by using the Systronics Spectrophotometer (Model -UV-VIS Systronics 117 series). Dissolved oxygen, total hardness, BOD, alkalinity, Calcium and Magnesium were measured following the methods outlined in APHA. ${ }^{14}$ For collection of phytoplankton, the plankton net of mesh size $20 \mu \mathrm{m}$ was used and the samples of phytoplankton were immediately preserved in water sample bottle containing $4 \%$ formalin solution for further analysis. Olympus $\mathrm{CH}$ $20 i$ Microscope was used for qualitative analysis. The density of phytoplankton was recorded in individuals per liter (ind..$^{-1}$ ) by using the Sedgwick Rafter counting cell. Primary taxonomic references used for the identification for phytoplankton were APHA'; Munshi et al. ${ }^{15}$ and Bellinger \& Sigee. ${ }^{16}$ Anova (analysis of Variance) was computed for assessing the significance level of seasonal variation in density of Phytoplankton. Multiple regression analysis of biological and environmental factors was performed. Canonical Correspondence Analysis (CCA) was also performed the using P Aleonotological Statistics (PAST) Software version 2.10.

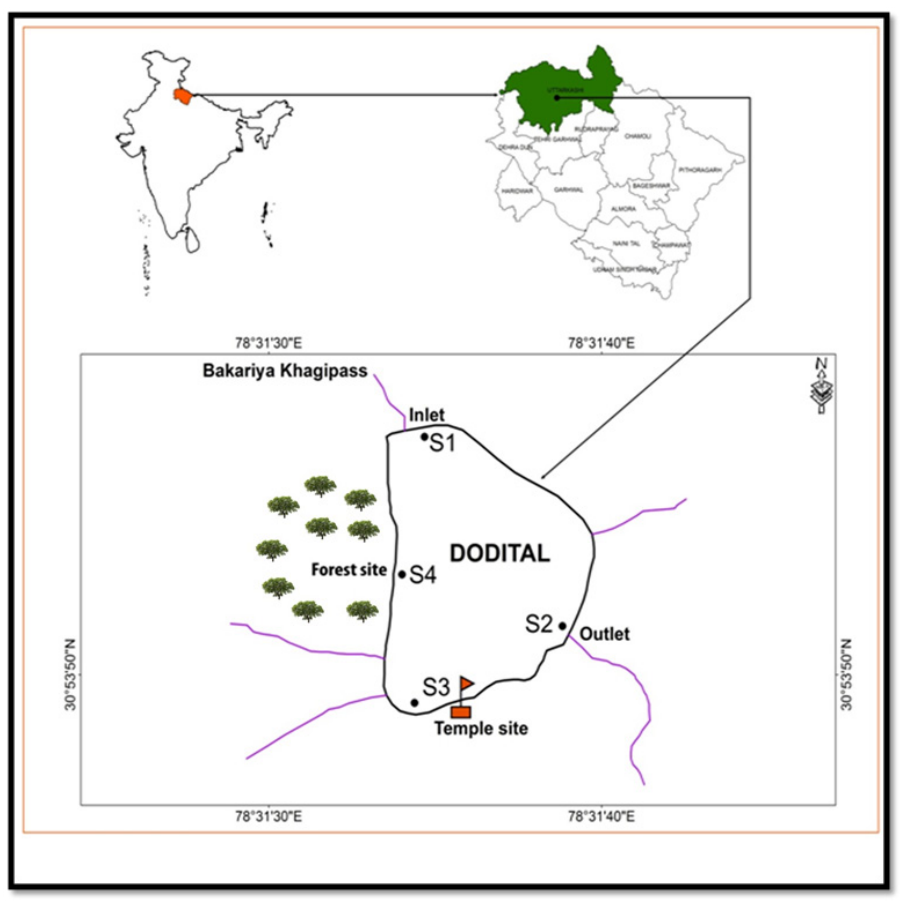

Figure I Location map of Dodi Tal with sampling sites.

\section{Results and discussion}

\section{Physico-chemical environmental variables}

High altitude wetlands of the Himalaya represent a relatively common ecosystem in mountain ranges in general; however, they remain less intensely studied than lowland wetlands, mainly because of their remoteness and the short summer open-water period. ${ }^{5}$ The analysis of physico-chemical parameters of Dodi Tal revealed that the air temperature $\left(12.70^{\circ} \mathrm{C}\right)$ was recorded in the month of May and minimum $\left(4.20^{\circ} \mathrm{C}\right)$ in the month of January. The same pattern was recorded in the water temperature. Turbidity was found to be maximum (1.79NTU) in the month of July and minimum (0.04NTU) in the month of January. Total Dissolved Solids (TDS) were found to be maximum (241 $\left.\mathrm{mg} \mathrm{l}^{-1}\right)$ in the month of August and minimum $\left(22.5 \mathrm{mg} \mathrm{1}^{-1}\right)$ in the month of December. This may be due to mixing if allochthonous materials in the lake during July-August (monsoon season). Conductivity of water in Dodi Tal was found to be maximum $\left(449 \mu \mathrm{Scm}^{-1}\right)$ in the month of August and minimum $\left(42.5 \mu \mathrm{Scm}^{-1}\right)$ in the month of December. Similar range of variation $(212 \pm 2.3 \mu \mathrm{S} / \mathrm{cm})$ was observed in Chandra Tal, Himachal Pradesh. ${ }^{9}$ Alkalinity of water in Dodi Tal was found to be maximum $\left(48.5 \mathrm{mg}^{-1}\right)$ in the month of July and minimum (26 $\left.\mathrm{m} \mathrm{l}^{-1}\right)$ in the month of November. Similar range of variation $\left(13 \mathrm{mg} / 1-37 \mathrm{mg} \mathrm{l}^{-1}\right.$ was recorded in Nilang Lake, Kashmir. ${ }^{17}$ Free $\mathrm{CO}_{2}$ of water in Dodi Tal was found to be maximum $(4.84 \mathrm{mg}$ $\left.\mathrm{1}^{-1}\right)$ in the month of July and minimum $\left(2.2 \mathrm{mg} \mathrm{l}^{-1}\right)$ in the month of October. Similar trend $\left(4.60-5.20 \mathrm{mg} \mathrm{l}^{-1}\right)$ was observed in two high altitude ponds, Badrinath, Uttarakhand. ${ }^{17}$ The dissolved oxygen of water in Dodi Tal was found to be maximum $\left(12.6 \mathrm{mg} \mathrm{l}^{-1}\right)$ in the month of January and minimum $\left(8.9 \mathrm{mg}^{-1}\right)$ in the month of September. Similar trend (9.0 $\mathrm{mg} \mathrm{l}^{-1}-9.6 \mathrm{mg} \mathrm{l}^{-1}$ ) was observed in Shesnag lake, Kashmir ${ }^{17}$ 6.85 $\mathrm{mg} \mathrm{l}^{-1}-13.21 \mathrm{mg} \mathrm{l}^{-1}$ in Prashar lake, Himachal Pradesh. ${ }^{7}$ $\mathrm{pH}$ of water in Dodi Tal was found to be maximum (7.84) in the month of July and minimum (6.79) in the month of September. these range of variation $(8.5 \pm 0.2$ to $9.1 \pm 0.1,8.6 \pm 0.2)$ was observed in Chandra Tal, Himachal Pradesh; ${ }^{9} 8.5 \pm 0.2$ to $9.1 \pm 0 ; 9.1 \pm 0.1$ in Suraj Tal and Sissu lake, Himachal Pradesh; ${ }^{9} 6.20$ to 8.20 in high altitude lake of Khumbu Valley. ${ }^{18,19}$ Chlorides of water in Dodi Tal were found to be maximum (14.2 $\mathrm{mg} \mathrm{l}^{-1}$ ) in the month of April and minimum $(4.26 \mathrm{mg}$ $\left.1^{-1}\right)$ in the month of August. Similar trend $\left(3.55 \pm 0.5 \mathrm{mg} \mathrm{l}^{-1}\right.$ to $56 \pm 2.5 \mathrm{mg}$ $\left.1^{-1}\right)$ was observed in Chandra Tal and Sissu Lake, Himachal Pradesh. ${ }^{9}$ Hardness of water of Dodi Tal was found to be maximum (38.8 mg $\left.\mathrm{l}^{-1}\right)$ in the month of July and minimum $\left(15.8 \mathrm{mg} \mathrm{l}^{-1}\right)$ in the month of December. Similar range of variation $(35 \mathrm{mg} / 1-37 \mathrm{mg} / \mathrm{l})$ was observed in Sheshnag Lake, Kashmir. ${ }^{17}$ Calcium concentration in Dodi Tal was found to be maximum $\left(7.21 \mathrm{mg} \mathrm{l}^{-1}\right)$ in the month of July and minimum $\left(4.16 \mathrm{mg} \mathrm{l}^{-1}\right)$ in the month of November. Magnesium concentration in Dodi Tal was found to be maximum $\left(5.22 \mathrm{mg} \mathrm{l}^{-1}\right)$ in the month of July and minimum $\left(1.27 \mathrm{mg}^{1^{-1}}\right)$ in the month of December. Similar range $\left(30.46 \pm 4.6 \mathrm{mg} \mathrm{l}^{-1}\right.$ to $\left.53 \pm 5.0 \mathrm{mg} \mathrm{l}^{-1}\right)$ of magnesium was recorded in Deepak Tal and $8.76 \pm 0.5 \mathrm{mg} \mathrm{l}^{-1}$ to $30 \pm 2.8 \mathrm{mg} \mathrm{l}^{-1}$ Sissu Lake, Himachal Pradesh. ${ }^{9}$ The concentration of nitrates of Dodi Tal was found to be maximum $\left(0.22 \mathrm{mg} \mathrm{l}^{-1}\right)$ in the month of July and minimum $(0.018 \mathrm{mg}$ $\left.1^{-1}\right)$ in the month of December. Similar range of variations $(0.38 \mathrm{mg}$ $\left.\mathrm{1}^{-1}-0.43 \mathrm{mg}^{-1}\right)$ was observed in two high altitude ponds, Badrinath, Uttarakhand, ${ }^{17} 0.29 \mathrm{mg}^{1-1}$ to $0.82 \mathrm{mg} \mathrm{l}^{-1}$ in high altitude lake of Khumbu , Imja Kola Valley. ${ }^{19}$ Phosphates were found to be maximum $(0.18 \mathrm{mg}$ $\left.1^{-1}\right)$ in the month of August and minimum $\left(0.015 \mathrm{mg} \mathrm{l}^{-1}\right)$ in the month of December. Similar range of variation $\left(0.05-0.08 \mathrm{mg} \mathrm{l}^{-1}\right)$ was found Nachiketatal, Uttarakhand. ${ }^{13}$ Sulphates were found to be maximum $\left(0.12 \mathrm{mg} \mathrm{l}^{-1}\right)$ in the month of August and minimum $\left(0.011 \mathrm{mg} \mathrm{l}^{-1}\right)$ in the month of December. Sodium concentration of Dodi Tal was found to be maximum $\left(1.19 \mathrm{mg} \mathrm{1}^{-1}\right)$ in the month of September and minimum $\left(0.43 \mathrm{mg} \mathrm{l}^{-1}\right)$ in the month of January. Potassium concentration of Dodi Tal was found to be maximum $\left(1.49 \mathrm{mg}^{-1}\right)$ in the month of September and minimum $\left(0.55 \mathrm{mg} \mathrm{l}^{-1}\right)$ in the month of January (Table 1).

\section{Phytoplankton community}

A total of 47 taxa belonging to 43 genera of phytoplankton were encounted during the present study. Phytoplankton were represented by Bacillariophyceae (20 genera), Chlorophyceae (16 genera), Cyanophyceae (4 genera) and Dinophyceae (3genera). Bacillariophyceae contributed $(47 \%)$ to the total phytoplankton, followed by Chlorophyceae (37\%), Cyanophyceae (9\%) and Dinophyceae (7\%) (Figure 2). The members of Bacillariophyceae (23) were represented by Cymbella cistuala Ehrenberg O.Kirchner, 1878, Diatoma vulgaris Bory 1824, fragilaria capucina Desmazières 
1830, Nitzschia recta Hantzsch ex Rabenhorst 1862, Synedra ulna Ehrenberg 1832, Synedra acus Kützing 1844, Tabellaria fenestrata (Lyngbye) Kützing 1844, Tabellaria flocculosa (Roth) Kützing 1844 (Figure 3). These species were also reported from Badrinath ponds, Uttarakhand. Bacillariophyceae was dominant among phytoplankton. ${ }^{18}$ Bacillariophyceae and Chlorophyceae were dominant groups in Nainital lake, Uttarakhand. ${ }^{11}$ Rawat \& Sharma ${ }^{20}$ reported 36 species of phytoplankton belonging to four groups, viz, Chlorophyceae, Bacillariophyceae, Dinophyceae and Cyanophycea from Deoria Tal, Uttarakhand. The most dominant species were Fragilaria sp., Navicula sp., Cyclotella sp., Ankistrodesmus falcatus, Pediastrum sp. Oscillatoria sp., Nitzschia sp., Cymbella sp. Closterium sp., Cladophora sp., Ulohtrix sp., Spirogyra sp., in high altitude wetland, Dodi Tal. These species were also reported from Kailash Lake, Kashmir; ${ }^{21}$ Badrinath ponds, Uttarakhand; ${ }^{22}$ Nainital, Uttarakhand;"11 Rewalsar lake, Himachal Pradesh;22 Wular lake, Kashmir. ${ }^{23}$ Anabena sp. and Ocillatoria sp. of Cynophyceae were also present in Dodi Tal. Similar species have been reported from Nanintal lake, Uttarakhand by Negi \& Rajput. ${ }^{11}$ Jindal et al. ${ }^{22}$ recorded from in Rewalsar lake, Himachal Pradesh; while Singh et al. ${ }^{9}$ reported these species from Chandra Tal, Suraj Tal, Deepak Tal and Sissu lake in Lahaul Spiti, Himachal Pradesh. Seasonally, the total phytoplankton were found to be maximum $\left(1270 \pm 315.00\right.$ ind. $\left.^{-1}\right)$ in autumn and minimum $\left(506.34 \pm 235.5\right.$ ind. $\left.1^{-1}\right)$ in monsoon. Seasonally, the density of Bacillariophyceae was found to be maximum (545.00 \pm 278.06 ind. $\left.1^{-1}\right)$ in summer season and minimum $\left(120.50 \pm 4.50\right.$ ind. $\left.1^{-1}\right)$ in monsoon (Figure 3). Chlorophyceae dwelling in Dodi Tal was found to be maximum $\left(731.00 \pm 88.00\right.$ ind..$\left.^{-1}\right)$ in autumn season and minimum $\left(169.67 \pm 8.81\right.$ ind. $\left.^{-1}\right)$ in summer season. Cyanophyceae dwelling in the Dodi Tal was found to be maximum $\left(42.50 \pm 10.50\right.$ ind. $\left.^{-1}\right)$ in monsoon season and minimum $\left(7.67 \pm 1.25\right.$ ind. $\left.1^{-1}\right)$ in winter season. Dinophyceae dwelling in the Dodi Tal was found to be maximum

Table I Seasonal variations in physico-chemical environmental variables of Dodi Tal during November 2014 to October 2016

\begin{tabular}{|c|c|c|c|c|c|}
\hline Environmental variables & Sites & Winter & Summer & Monsoon & Autumn \\
\hline \multirow{4}{*}{ Air temp $\left({ }^{\circ} \mathrm{C}\right)$} & $\mathrm{S}^{\prime}$ & $7.67 \pm 2.368$ & $9.73 \pm 2.444$ & $12.05 \pm 0.550$ & $10.60 \pm 0.700$ \\
\hline & $\mathrm{S}^{2}$ & $7.73 \pm 2.409$ & $9.83 \pm 2.370$ & $12.15 \pm 0.550$ & $10.75 \pm 0.650$ \\
\hline & $\mathrm{S}^{3}$ & $7.77 \pm 2.409$ & $9.90 \pm 2.342$ & $12.20 \pm 0.500$ & $10.90 \pm 0.600$ \\
\hline & $\mathrm{S}^{4}$ & $7.87 \pm 2.409$ & $9.97 \pm 2.316$ & $12.30 \pm 0.500$ & $11.00 \pm 0.600$ \\
\hline \multirow{4}{*}{ Water temp. $\left({ }^{\circ} \mathrm{C}\right)$} & $S^{\prime}$ & $6.37 \pm 2.819$ & $8.60 \pm 2.998$ & $10.35 \pm 1.050$ & $9.40 \pm 1.000$ \\
\hline & $S^{2}$ & $6.43 \pm 2.811$ & $8.80 \pm 2.922$ & $10.45 \pm 1.050$ & $9.55 \pm 0.950$ \\
\hline & $\mathrm{S}^{3}$ & $6.57 \pm 2.798$ & $8.90 \pm 2.922$ & $10.55 \pm 1.050$ & $9.75 \pm 0.950$ \\
\hline & $\mathrm{S}^{4}$ & $6.47 \pm 2.923$ & $9.00 \pm 2.922$ & $10.65 \pm 1.050$ & $9.85 \pm 0.950$ \\
\hline \multirow{4}{*}{ Turbidity (NTU) } & $S^{\prime}$ & $0.14 \pm 0.056$ & $0.93 \pm 0.184$ & $1.425 \pm 0.155$ & $1.03 \pm 0.065$ \\
\hline & $S^{2}$ & $0.15 \pm 0.059$ & $0.94 \pm 0.188$ & $1.44 \pm 0.160$ & $1.07 \pm 0.045$ \\
\hline & $S^{3}$ & $0.17 \pm 0.062$ & $0.97 \pm 0.184$ & $1.26 \pm 0.045$ & $1.13 \pm 0.040$ \\
\hline & $\mathrm{S}^{4}$ & $0.21 \pm 0.052$ & $1.01 \pm 0.184$ & $1.33 \pm 0.060$ & $1.22 \pm 0.035$ \\
\hline \multirow{4}{*}{ TDS (mg l-1) } & $S^{\prime}$ & $36.23 \pm 13.996$ & III. $.70 \pm 57.874$ & $165 \pm 47.00$ & $61.70 \pm 36.500$ \\
\hline & $\mathrm{S}^{2}$ & $36.73 \pm 13.934$ & $1 \mid 2.90 \pm 58.267$ & $168.50 \pm 47.50$ & $62.30 \pm 36.500$ \\
\hline & $S^{3}$ & $37.07 \pm 13.995$ & $\mid 14.30 \pm 59.114$ & $170.50 \pm 47.50$ & $62.40 \pm 36.500$ \\
\hline & $S^{4}$ & $37.37 \pm 14.196$ & | | $9.07 \pm 55.888$ & $172.00 \pm 47.00$ & $62.65 \pm 36.450$ \\
\hline \multirow{4}{*}{ Conductivity $\left(\mu \mathrm{Scm}^{-1}\right)$} & $S^{\prime}$ & $64.00 \pm 15.513$ & $|58.83 \pm 83.60|$ & $363 \pm 70.00$ & $82.10 \pm 21.900$ \\
\hline & $\mathrm{S}^{2}$ & $64.47 \pm 15.696$ & $159.97 \pm 83.879$ & $366.50 \pm 70.50$ & $84.95 \pm 24.050$ \\
\hline & $\mathrm{S}^{3}$ & $64.67 \pm 15.618$ & $161.20 \pm 84.035$ & $368.50 \pm 70.50$ & $86.70 \pm 25.300$ \\
\hline & $S^{4}$ & $64.45 \pm \mid 4.849$ & $\mid 63.27 \pm 85.580$ & $369.50 \pm 70.50$ & $88.45 \pm 26.550$ \\
\hline
\end{tabular}

Citation: Sharma RC, Singh S. Water quality and phytoplankton diversity of high altitude wetland, Dodi Tal of Garhwal Himalaya, India. Biodiversity Int J. 2018;2(6):484-493. DOI: I0.15406/bij.2018.02.00103

$\left(109.00 \pm 50.64\right.$ ind. $\left.1^{-1}\right)$ in summer season and minimum $(33.67 \pm 4.99$ ind. $\left.1^{-1}\right)$ in winter season (Figure 3). A highly significant $(\mathrm{F}=14.58$; $\mathrm{p}=143 \mathrm{E}^{-08}$ ) seasonal variation in density of phytoplankton of Dodi Tal was recorded.

However, if the comparison of the phytoplankton diversity of high altitude wetland Dodi Tal is made with the phytoplankton diversity of low level wetlands of India is made, it is different in diversity, density and seasonal variability. Maximum diversity in lowland wetlands has been reported in summer season and minimum in winter season. ${ }^{23-25}$ Presence in the species of phytoplankton was also different in the lowland wetlands in comparison to the high altitude wetland Dodi Tal. This may be due to the difference in seasonal variations in the physico-chemical variables of the water quality in Dodi Tal with that of the lowland wetlands.

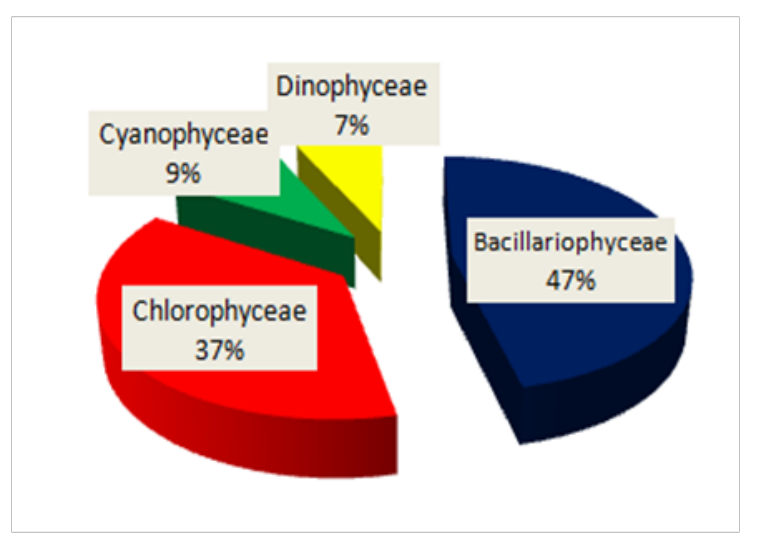

Figure 2 Percentage composition of phytoplankton in high altitude wetland, Dodi Tal. 


\begin{tabular}{|c|c|c|c|c|c|}
\hline \multirow{4}{*}{ Alkalinity (mg..-1) } & $S^{\prime}$ & $32.50 \pm 7.427$ & $35.33 \pm 7.587$ & $44.75 \pm 1.750$ & $33.25 \pm 3.250$ \\
\hline & $\mathrm{S}^{2}$ & $33.00 \pm 7.427$ & $35.83 \pm 7.587$ & $45.25 \pm 1.750$ & $33.75 \pm 3.250$ \\
\hline & $S^{3}$ & $33.50 \pm 7.427$ & $36.33 \pm 7.587$ & $45.75 \pm 1.750$ & $34.25 \pm 3.250$ \\
\hline & $\mathrm{S}^{4}$ & $33.83 \pm 7.192$ & $36.83 \pm 7.587$ & $46.00 \pm 1.500$ & $34.50 \pm 3.000$ \\
\hline \multirow{4}{*}{ Free $\mathrm{CO} 2\left(\mathrm{mg} \mathrm{l}^{-1}\right)$} & $S^{\prime}$ & $2.93 \pm 0.452$ & $3.52 \pm 0.180$ & $3.74 \pm 0.660$ & $2.75 \pm 0.110$ \\
\hline & $\mathrm{S}^{2}$ & $2.93 \pm 0.452$ & $3.52 \pm 0.180$ & $3.74 \pm 0.660$ & $2.75 \pm 0.110$ \\
\hline & $S^{3}$ & $3.01 \pm 0.549$ & $3.67 \pm 0.104$ & $3.85 \pm 0.770$ & $2.75 \pm 0.110$ \\
\hline & $\mathrm{S}^{4}$ & $3.01 \pm 0.549$ & $3.67 \pm 0.104$ & $3.85 \pm 0.770$ & $2.75 \pm 0.110$ \\
\hline \multirow{4}{*}{ Dissolved oxygen (mg..-1) } & $S^{\prime}$ & $12.07 \pm 0.525$ & $10.40 \pm 0.163$ & $9.9 \pm 0.100$ & $10.40 \pm 0.400$ \\
\hline & $S^{2}$ & $11.83 \pm 0.544$ & $10.27 \pm 0.125$ & $9.75 \pm 0.050$ & $10.20 \pm 0.400$ \\
\hline & $S^{3}$ & $|1.57 \pm 0.59|$ & $10.03 \pm 0.170$ & $9.65 \pm 0.050$ & $10.05 \pm 0.350$ \\
\hline & $S^{4}$ & $11.33 \pm 0.544$ & $9.87 \pm 0.205$ & $9.55 \pm 0.050$ & $9.95 \pm 0.350$ \\
\hline \multirow{4}{*}{ B.O.D } & $S^{\prime}$ & $0.33 \pm 0.094$ & $0.33 \pm 0.094$ & $0.7 \pm 0.100$ & $0.70 \pm 0.100$ \\
\hline & $\mathrm{S}^{2}$ & $0.33 \pm 0.094$ & $0.40 \pm 0.082$ & $0.85 \pm 0.050$ & $0.75 \pm 0.050$ \\
\hline & $S^{3}$ & $0.40 \pm 0.082$ & $0.50 \pm 0.082$ & $1.00 \pm 0.100$ & $0.85 \pm 0.050$ \\
\hline & $S^{4}$ & $0.40 \pm 0.082$ & $0.53 \pm 0.094$ & $1.00 \pm 0.100$ & $0.85 \pm 0.050$ \\
\hline \multirow{4}{*}{$\mathrm{pH}$} & $S^{\prime}$ & $7.65 \pm 0.173$ & $7.30 \pm 0.243$ & $7.72 \pm 0.110$ & $7.04 \pm 0.075$ \\
\hline & $\mathrm{S}^{2}$ & $7.64 \pm 0.163$ & $7.27 \pm 0.232$ & $7.68 \pm 0.110$ & $7.01 \pm 0.090$ \\
\hline & $S^{3}$ & $7.62 \pm 0.170$ & $7.29 \pm 0.239$ & $7.7 I \pm 0.110$ & $7.02 \pm 0.075$ \\
\hline & $S^{4}$ & $7.57 \pm 0.184$ & $7.25 \pm 0.24 I$ & $7.69 \pm 0.105$ & $6.95 \pm 0.030$ \\
\hline \multirow{4}{*}{ Chlorides ( $\mathrm{mg} \mathrm{^{-1 } )}$} & $S^{\prime}$ & $5.68 \pm 1.159$ & $6.15 \pm 0.669$ & $9.23 \pm 0.710$ & $5.68 \pm 1.420$ \\
\hline & $\mathrm{S}^{2}$ & $6.15 \pm 0.669$ & $6.15 \pm 0.669$ & $9.23 \pm 0.710$ & $5.68 \pm 1.420$ \\
\hline & $S^{3}$ & $6.63 \pm 1.339$ & $7.10 \pm 2.008$ & $10.65 \pm 0.710$ & $5.68 \pm 1.420$ \\
\hline & $\mathrm{S}^{4}$ & $6.63 \pm 1.339$ & $7.10 \pm 2.008$ & $12.07 \pm 0.710$ & $6.39 \pm 2.130$ \\
\hline \multirow{4}{*}{ Total Hardness(mg l-1) } & $S^{\prime}$ & $21.07 \pm 7.173$ & $25.47 \pm 5.342$ & $35.5 \pm 2.700$ & $22.30 \pm 2.500$ \\
\hline & $\mathrm{S}^{2}$ & $21.13 \pm 7.267$ & $25.47 \pm 5.342$ & $35.65 \pm 2.750$ & $22.30 \pm 2.500$ \\
\hline & $S^{3}$ & $21.20 \pm 7.224$ & $25.47 \pm 5.342$ & $35.90 \pm 2.700$ & $22.30 \pm 2.500$ \\
\hline & $\mathrm{S}^{4}$ & $21.33 \pm 7.130$ & $25.53 \pm 5.429$ & $36.00 \pm 2.800$ & $22.30 \pm 2.500$ \\
\hline \multirow{4}{*}{ Calcium $\left(\mathrm{mg} \mathrm{l}^{-1}\right)$} & $S^{\prime}$ & $4.70 \pm 0.590$ & $5.10 \pm 0.512$ & $5.89 \pm 0.760$ & $4.73 \pm 0.325$ \\
\hline & $\mathrm{S}^{2}$ & $4.72 \pm 0.628$ & $5.15 \pm 0.548$ & $5.97 \pm 0.760$ & $4.8 I \pm 0.325$ \\
\hline & $S^{3}$ & $4.72 \pm 0.628$ & $5.18 \pm 0.560$ & $6.05 \pm 0.760$ & $4.8 I \pm 0.325$ \\
\hline & $\mathrm{S}^{4}$ & $4.72 \pm 0.628$ & $5.2 \mathrm{I} \pm 0.574$ & $6.13 \pm 0.760$ & $4.8 I \pm 0.325$ \\
\hline \multirow{4}{*}{ Magnesium ion (mg l-1) } & $S^{\prime}$ & $2.27 \pm 1.394$ & $3.10 \pm 1.384$ & $5.065 \pm 0.195$ & $2.56 \pm 0.410$ \\
\hline & $\mathrm{S}^{2}$ & $2.27 \pm 1.394$ & $3.07 \pm 1.387$ & $5.06 \pm 0.205$ & $2.5 I \pm 0.410$ \\
\hline & $S^{3}$ & $2.27 \pm 1.394$ & $3.05 \pm 1.368$ & $5.07 \pm 0.195$ & $2.51 \pm 0.410$ \\
\hline & $S^{4}$ & $2.29 \pm 1.381$ & $3.05 \pm 1.368$ & $5.04 \pm 0.220$ & $2.5 I \pm 0.410$ \\
\hline \multirow{4}{*}{ Nitrates $\left(\mathrm{mg} \mathrm{l}^{-1}\right)$} & $S^{\prime}$ & $0.02 \pm 0.004$ & $0.08 \pm 0.009$ & $0.10 \pm 0.011$ & $0.08 \pm 0.011$ \\
\hline & $\mathrm{S}^{2}$ & $0.03 \pm 0.005$ & $0.09 \pm 0.008$ & $0.13 \pm 0.010$ & $0.08 \pm 0.013$ \\
\hline & $S^{3}$ & $0.03 \pm 0.004$ & $0.10 \pm 0.011$ & $0.17 \pm 0.006$ & $0.10 \pm 0.023$ \\
\hline & $S^{4}$ & $0.04 \pm 0.007$ & $0.13 \pm 0.054$ & $0.22 \pm 0.010$ & $0.12 \pm 0.040$ \\
\hline \multirow{4}{*}{ Phosphates $\left(\mathrm{mg} \mathrm{l}^{-1}\right)$} & $S^{\prime}$ & $0.02 \pm 0.004$ & $0.07 \pm 0.010$ & $0.096 \pm 0.001$ & $0.07 \pm 0.009$ \\
\hline & $S^{2}$ & $0.03 \pm 0.005$ & $0.09 \pm 0.008$ & $0.13 \pm 0.010$ & $0.08 \pm 0.013$ \\
\hline & $S^{3}$ & $0.02 \pm 0.003$ & $0.08 \pm 0.010$ & $0.09 \pm 0.000$ & $0.07 \pm 0.009$ \\
\hline & $\mathrm{S}^{4}$ & $0.03 \pm 0.003$ & $0.08 \pm 0.005$ & $0.09 \pm 0.000$ & $0.08 \pm 0.007$ \\
\hline
\end{tabular}




\begin{tabular}{|c|c|c|c|c|c|}
\hline \multirow{4}{*}{ Sulphates $\left(\mathrm{mg} \mathrm{l}^{-1}\right)$} & $S^{\prime}$ & $0.01 \pm 0.004$ & $0.05 \pm 0.022$ & $0.087 \pm 0.002$ & $0.06 \pm 0.009$ \\
\hline & $\mathrm{S}^{2}$ & $0.01 \pm 0.004$ & $0.06 \pm 0.02$ & $0.09 \pm 0.002$ & $0.06 \pm 0.010$ \\
\hline & $S^{3}$ & $0.02 \pm 0.004$ & $0.07 \pm 0.020$ & $0.09 \pm 0.000$ & $0.07 \pm 0.012$ \\
\hline & $S^{4}$ & $0.02 \pm 0.003$ & $0.07 \pm 0.009$ & $0.09 \pm 0.000$ & $0.07 \pm 0.010$ \\
\hline \multirow{4}{*}{ Sodium $\left(\mathrm{mg} \mathrm{l}^{-1}\right)$} & $S^{\prime}$ & $0.77 \pm 0.226$ & $0.80 \pm 0.127$ & $0.675 \pm 0.135$ & $0.76 \pm 0.200$ \\
\hline & $S^{2}$ & $0.81 \pm 0.227$ & $0.85 \pm 0.127$ & $0.80 \pm 0.075$ & $0.81 \pm 0.180$ \\
\hline & $S^{3}$ & $0.94 \pm 0.142$ & $0.95 \pm 0.101$ & $0.95 \pm 0.090$ & $0.91 \pm 0.170$ \\
\hline & $S^{4}$ & $1.07 \pm 0.116$ & $1.06 \pm 0.087$ & $1.02 \pm 0.070$ & $0.95 \pm 0.130$ \\
\hline \multirow{4}{*}{ Potassium $\left(\mathrm{mg} \mathrm{l}^{-1}\right)$} & $S^{\prime}$ & $0.86 \pm 0.207$ & $0.92 \pm 0.116$ & $0.88 \pm 0.010$ & $0.94 \pm 0.140$ \\
\hline & $S^{2}$ & $0.97 \pm 0.119$ & $1.02 \pm 0.084$ & $0.97 \pm 0.020$ & $0.98 \pm 0.165$ \\
\hline & $S^{3}$ & $1.04 \pm 0.114$ & $1.11 \pm 0.078$ & $1.14 \pm 0.010$ & $1.07 \pm 0.140$ \\
\hline & $\mathrm{S}^{4}$ & $1.18 \pm 0.050$ & $1.36 \pm 0.062$ & $1.45 \pm 0.010$ & $1.11 \pm 0.115$ \\
\hline
\end{tabular}

Table 2 Seasonal variations in diversity index (Shannon-Wiener, 1964) for phytoplankton of high altitude wetland, Dodi Tal, Garhwal Himalaya for the period November 2014-October 2016

\begin{tabular}{llllll}
\hline Sites & Winter & Spring & Summer & Monsoon & Autumn \\
\hline $\mathbf{2 0 1 4 - 2 0 1 5}$ & & & & & \\
$\mathrm{S}^{1}$ & 3.73 & $\mathrm{NA}$ & 4.07 & 3.59 & 4.08 \\
$\mathrm{~S}^{2}$ & 3.75 & $\mathrm{NA}$ & 4.07 & 3.6 & 4.08 \\
$\mathrm{~S}^{3}$ & 3.78 & $\mathrm{NA}$ & $4.1 \mathrm{I}$ & 3.67 & 4.06 \\
$\mathrm{~S}^{4}$ & $3.8 \mathrm{I}$ & $\mathrm{NA}$ & $4.1 \mathrm{I}$ & 3.67 & 4.06 \\
$\bar{x}$ Diversity Index & 3.77 & $\mathrm{NA}$ & 4.09 & 3.63 & 4.07 \\
$\mathbf{2 0}$ I 5-20I6 & & & & & \\
$\mathrm{S}^{1}$ & 3.78 & $\mathrm{NA}$ & $4.1 \mathrm{I}$ & 3.65 & 4.1 \\
$\mathrm{~S}^{2}$ & 3.78 & $\mathrm{NA}$ & 4.12 & 3.65 & 4.1 \\
$\mathrm{~S}^{3}$ & $3.8 \mathrm{NA}$ & 4.18 & 3.67 & 4.1 \\
$\mathrm{~S}^{4}$ & 3.34 & $\mathrm{NA}$ & $3.9 \mathrm{I}$ & 3.22 & $3.4 \mathrm{I}$ \\
$\bar{x}$ Diversity Index & 3.68 & $\mathrm{NA}$ & 4.08 & 3.55 & 3.93 \\
\hline
\end{tabular}

\section{Shannon -Wiener diversity of phytoplankton}

Shannon Wiener Diversity index of phytoplankton can be considered as an important index for assessing the water quality of the wetlands. The Shannon Wiener diversity index calculated for phytoplankton of Dodi Tal has been presented in Table 2. For Indian high altitude wetlands, the Shannon-Wiener diversity index greater than $(>4)$ is considered as clean water; between 3-4 is mildly polluted water; and less than $2(<2)$ is heavily polluted water. ${ }^{27}$ Since, the Shannon-Wiener diversity index in the present study ranged between 3.55-4.09 (Table 2). Therefore, this water body falls in the diversity index $(>4)$, which means the clean water. So the water of the high altitude lake, Dodi Tal is clean and potable. Similar findings were reported (3.18-3.31) from Prashar Lake, Himachal Pradesh. ${ }^{28}$ Sharma \& Tiwari $^{13}$ reported 3.75-3.95 diversity index for Nachiketa Tal, Uttarakhand.

Liner regression was calculated between density of phytoplankton density and physico chemical parameters (Figure 4). Regression plot calculated between physico-chemical parameters and diversity of phytoplankton revealed that the phytoplankton density has position correlation with air temperature $\left(\mathrm{y}=4.705 \mathrm{x}+677.4, \mathrm{R}^{2}=0.01\right.$, where $\mathrm{y}=$ phytoplankton density); water temperature $(\mathrm{y}=5.754 \mathrm{x}$ $\left.+674.8, \quad \mathrm{R}^{2}=0.002\right), \quad \mathrm{BOD} \quad\left(\mathrm{y}=122.8 \mathrm{x}+653.4, \quad \mathrm{R}^{2}=0.006\right)$, nitrates $\left(y=89.16 x+731.8, \quad R^{2}=0.00\right.$, and phosphates $(y=77.24 x+731.8$, $\mathrm{R}^{2}=0.00$ ). However, the density of phytoplankton has negative correlation with turbidity $\left(\mathrm{y}=-83.21 \mathrm{x}-653.2, \mathrm{R}^{2}=0.012\right)$, sulphates $\left(y=-1639 x+625.7, R^{2}=0.021\right)$. TDS $\left(y=-2.098 x+920.00, R^{2}=0.150\right)$, conductivity $\left(\mathrm{y}=-1.295 \mathrm{x}+930.1, \quad \mathrm{R}^{2=} 0.0 .203\right)$, alkalinity $\quad(\mathrm{y}=-$ $\left.27.32 \mathrm{x}+1706, \mathrm{R}^{2}=0.337\right)$, free $\mathrm{CO}_{2}\left(\mathrm{y}=-295.1 \mathrm{x}+1678, \mathrm{R}^{2}=0.255\right)$ and dissolved oxygen $\left(\mathrm{y}=-114.2 \mathrm{x}+1920, \mathrm{R}^{2}=0.077\right)$ (Figure 4).

Multiple regressions computed between phytoplankton density and physico- chemical parameter were presented in Table 5. Phytoplankton density had negative correlation with TDS (F value $=1.414$, $\mathrm{t}$ stat $=-$ $1.189)$, alkalinity $(\mathrm{F}$ value $=4.083, \mathrm{t}$ stat $=-2.021)$, total dissolved solids $(\mathrm{F}$ value $=0.675, \mathrm{t}$ stat $=-0.822), \mathrm{pH}(\mathrm{F}$ value $=5.354$, $\mathrm{t}$ stat $=-2314)$ and chlorides ( $\mathrm{F}$ value $=0.298$, $\mathrm{t}$ stat $=-0.546$ ). However, the density of phytoplankton had positive correlation with water temperature $(\mathrm{F}$ value $=0.0158, \mathrm{t}$ stat $=0.126)$, tubidity $(\mathrm{F}$ value $=0.105$, $\mathrm{t}$ stat $=0.324)$, air temperature $(\mathrm{F}$ value $=0.0085$, $\mathrm{t}$ stat $=0.092)$, phosphates ( $\mathrm{F}$ value $=0.0005$, t stat $=0.022$ ) and nitrates (F value $=0.001$, t stat $=0.030$ ).

Canonical Correspondence Analysis (CCA) method was also used to determine the effect of physico- chemical parameters on phytoplankton taxa. The arrow length indicates the importance of variable and shows the positive or negative correlation with axis. ${ }^{1,9}$ CCA has been drawn between 19 physico- chemical parameters and 
47 phytoplankton genera. At the sampling site $\mathrm{S}_{1}$, Eigen value for axis $1(0.163)$ explained $37.51 \%$ correlation; and axis $2(0.090)$ explained $(20.71 \%)$ correlation between phytoplankton genera and physicochemical parameters. Dissolved oxygen affects the distribution of Gomphonema spp. Amphora spp., Diatoma vulgaris, Achanthes sp., Frustulia spp. which had strong positive correlation with axis 2. The distribution of Navicula spp. and Syndra acus was affected by chlorides and it had negative correlation with Axis 1. Alkalinity, total hardness, conductivity, Magnesium and Calcium affect the distribution of Ulothrix zonata, Ulothrix tenerrima, Spirogyra sp. which had strong negative correlation with axis 1 . These parameters are favourable for the growth of these phytoplankton species. Free $\mathrm{CO}_{2}$ and $\mathrm{pH}$ have negative correlation with Axis 1 and affect some phytoplankton genera including Spirulina sp., Oscillatoria sp.,
Anabena sp., Lyngbya sp., Nitzschia recta (Figure 5).

At $\mathrm{S}_{2}$, the Eigen value (0.159) explained $37.32 \%$ at axis 1 and Eigen value of axis $2(0.089)$ explained $20.92 \%$ correlation between physico-chemical parameters and phytoplankton genera. Distribution of Gomphonema spp. Amphora spp., Diatoma vulgaris, Frustulia spp. had strong positive correlation with axis 2 and affected by dissolved oxygen. Alkalinity, total hardness, Magnesium, Calcium, TDS, conductivity, and Calcium are favourable for the growth of Ulothrix zonata and Spirogyra sp. which have strong negative correlation with axis 1 . Even water temperature has a major role for the growth of the Closterium spp. which has negative correlation with axis 2 . Distribution of Oscillatoria sp., Anabena sp., and Nitzschia recta were affected by $\mathrm{pH}$ and free $\mathrm{CO}_{2}$ which have strong negative correlation with Axis 1 at $\mathrm{S}_{2}$ (Figure 6).

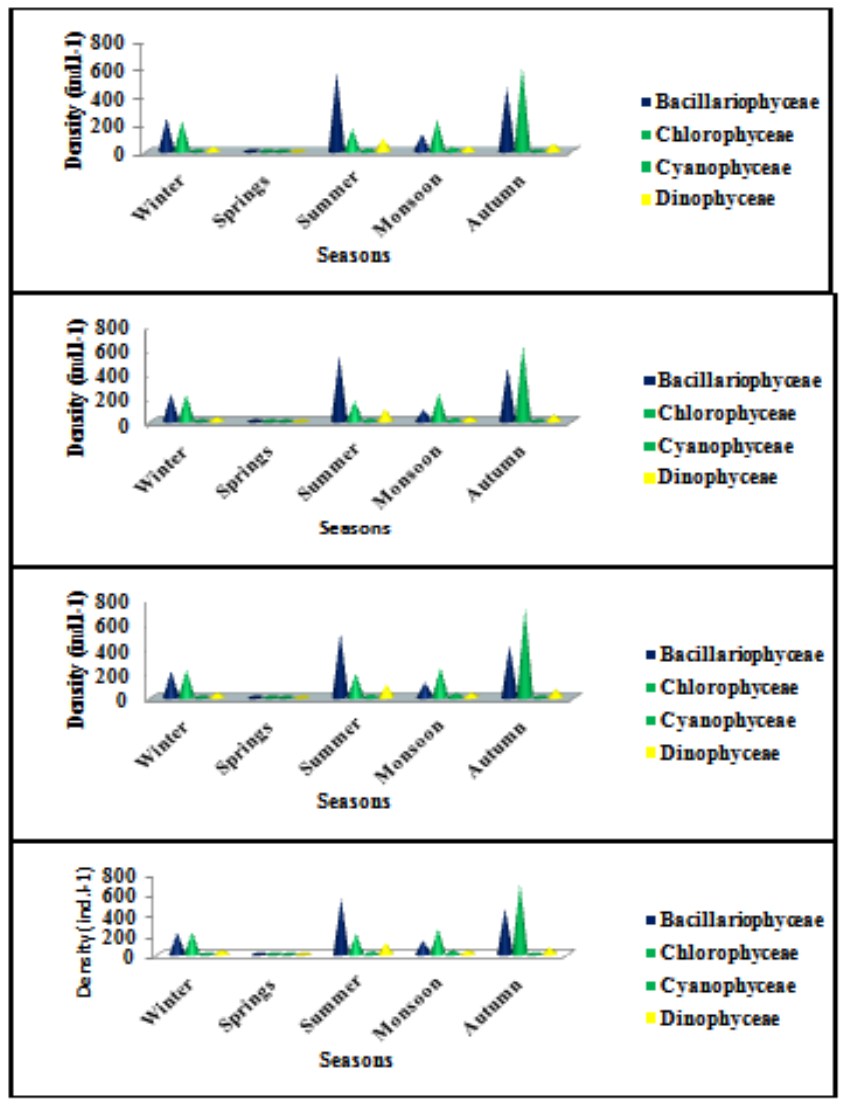

Figure 3 Seasonal variations in density of phytoplankton at all the four sites (SI-S4) of Dodi Tal.

While, at $\mathrm{S}_{3}$, axis 1 has Eigen value $(0.153)$ which explained $37.25 \%$ relationship between physiochemical parameters and phytoplankton community and Eigen value of axis 2 (0.088) explained $21.55 \%$ correlation between physico-chemical parameters and phytoplankton. Distribution of Zygnema spp was affected by Potassium and turbidity which had negative correlation with Axis 2. Sulphates affect the distribution of Xanthidium antilopaeum, water temperature and Sodium affect Closterium spp., and Mougeotia spp; Air temperature affect Volvox aureas and Pandorina morum, which has negative correlation with Axis 2. $\mathrm{pH}$ had negative correlation with Axis 1 and affect the growth of Spirulina sp. Alkalinity, total hardness and Magnesium affect Ulothrix zonata, Spirogyra spp., and Ulothrix tenerrima (Figure 7).

Whereas, at $\mathrm{S}_{4}$ site, axis 1 has Eigen value (0.146) explained $37.53 \%$ relationship between physiochemical parameters and phytoplankton community and Eigen value of axis 2 (0.082) explained $21.05 \%$ correaltion between physico-chemical parameters and phytoplankton. Dissolved oxygen and chlorides have negative correlation with phytoplankton genera at $\mathrm{S}_{4}$ on axis 1 . These parameters are favourable for the growth of these phytoplankton genera like Peridium cinctum, fragilaria capucina, Tabellaria fenestrate, Synedra acus, and Gomphonema spp. Alkalinity, conductivity, total hardness, $\mathrm{pH}$ and Magnesium affect the phytoplankton genera like Ulothrix tenerrima and Spirogyra sp on axis 1. But these parameters have shown the key role for survival of these phytoplankton species. $\mathrm{pH}$ and free $\mathrm{CO}_{2}$ have strong negative correlation with axis 1 affecting the distribution of Anabena spp. Spirulina sp. Physiochemical parameters did not show any effect on Oocystis spp. at $\mathrm{S}_{4}$ on axis 1 (Figure 8). 


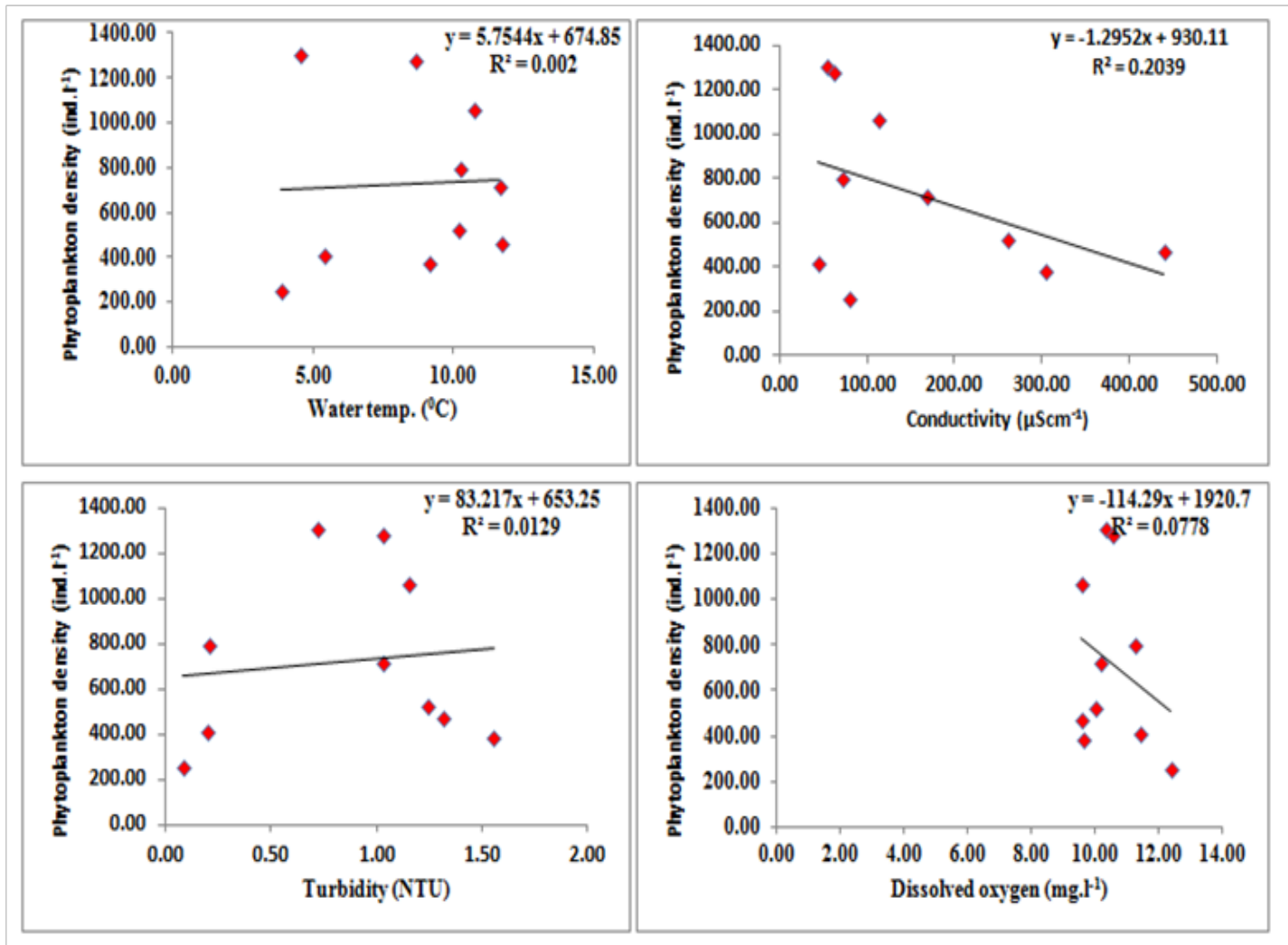

Figure 4 Regression analyses calculated between phytoplankton density and physicochemical parameters of Dodi Tal during the period from November 2015- October 2016.

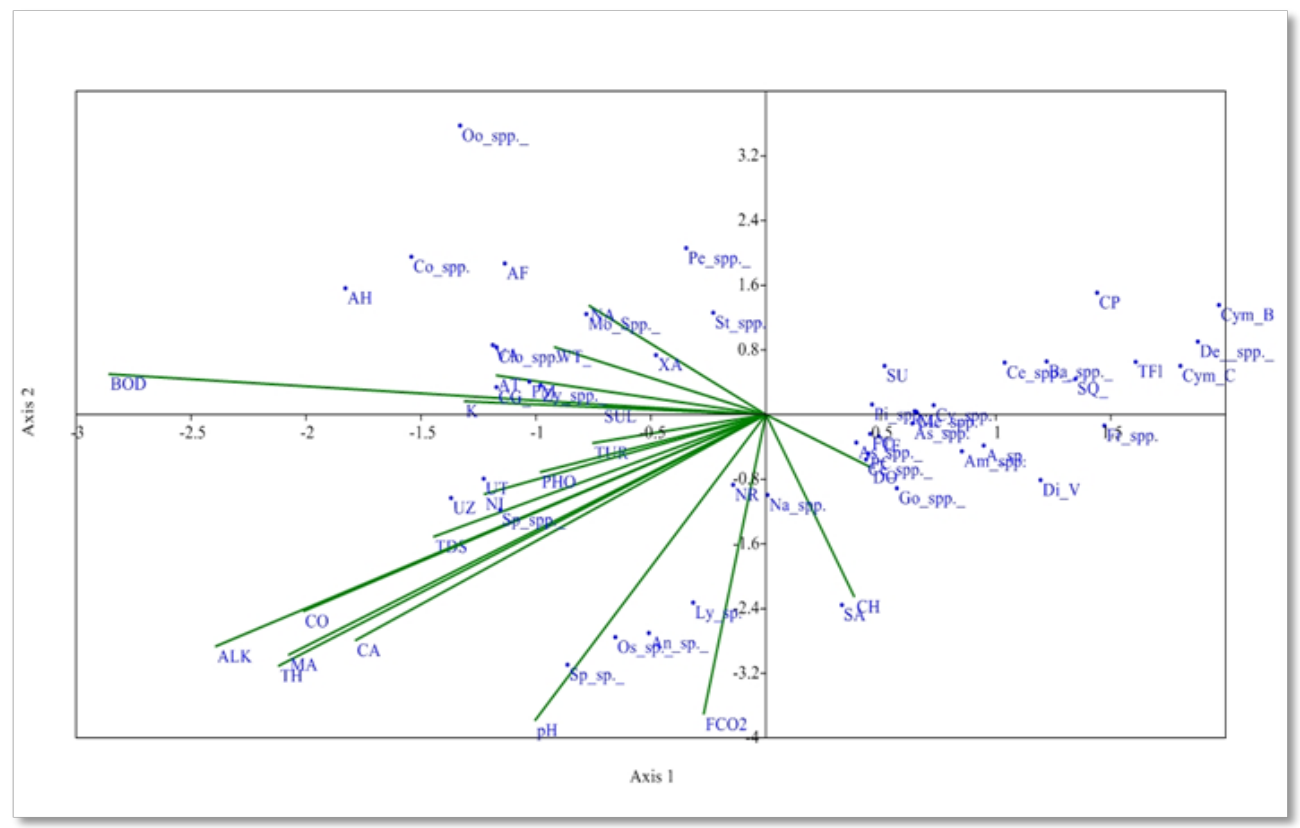

Figure 5 CCA biplot at $\mathrm{S}_{1}$ between physiochemical parameters and phytoplankton species (Physico-chemical parameters, AT, Air temperature; WT, Water temperature ;TUR, Turbidity ; TDS, Total Dissolved Solids; CO, Conductivity ;ALK , Alkalinity; FCO, Free CO2; DO, Dissolved Oxygen BOD, Biological Oxygen Demand; pH,; CH, Chlorides; TOH, Total hardness; CA, Calcium ; MA, Magnesium; NI, Nitrates; PHO, Phosphates; SUL, Sulphates; NA, Sodium; K, Potassium); (Phytoplankton species A sp , Achananthes sp.; Am spp , Amphora spp, As spp, Asterionella spp.; Ba spp, Bacillaria spp; Ce spp, Ceratoneis spp; CL, Closteriopsis longissima; CP, Cocconeis placentula; Cy spp., Cyclotella spp. Cym B, Cymbella bengalensis; Cym c, Cymbella cistuala; De spp, Denticula spp.; Di V, Diatoma vulgaris; fr spp, Frustulia spp.. FC, fragilaria capucina; Go, Gomphonema spp; Me, Melosira spp; Na, Navicula spp.; NR, Nitzschia recta; Pi spp, Pinnularia spp.. SU, Synedra ulna; SA; Synedra acus TF Tabellaria fenestrata; TFI, Tabellaria flocculosa; AH, Actinastrum hantzschii; AF, c;CG, Cladophora glomerata; Co spp, Cosmarium spp.; Clo spp, Closterium spp.; Mo spp, Mougeotia Spp.. Oo spp, Oocystis spp.. PM, Pandorina morum; Pe spp, Pediastrum spp. SQ, Scenedesmus quadricauda; Sp spp, Spirogyra spp.. St spp, Staurastrum spp..; UZ, Ulothrix zonata; UT, Ulothrix tenerrima;VA, Volvox aureas; XA, Xanthidium antilopaeum; Zy spp, Zygnema spp.; An sp, Anabaena sp...; Ly sp, Lyngbya sp..; Os sp, Oscillatoria sp..; Sp sp, Spirulina sp..; Ce spp, Ceratium spp.. PC, Peridinium cinctum;As spp. Asterocystis spp.). 


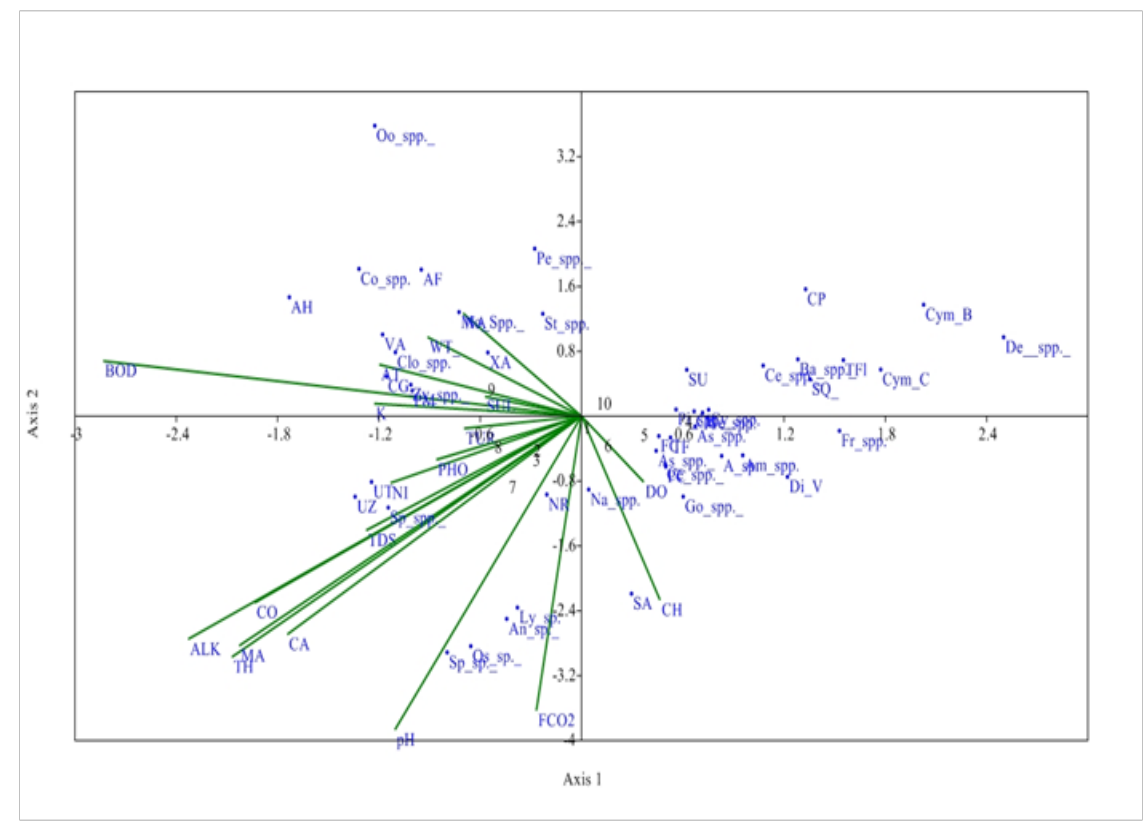

Figure 6 CCA biplot at $S_{2}$ between physiochemical parameters and phytoplankton species (Physico-chemical parameters, AT, Air temperature; WT, Water temperature ;TUR, Turbidity ; TDS, Total Dissolved Solids; CO, Conductivity ;ALK ,Alkalinity; FCO, Free $\mathrm{CO}_{2}$; DO, Dissolved Oxygen BOD, Biological Oxygen Demand ; pH,; CH, Chlorides; TOH, Total hardness ; CA, Calcium ; MA, Magnesium; NI, Nitrates; PHO, Phosphates; SUL, Sulphates; NA, Sodium ; K, Potassium ); (Phytoplankton species A sp , Achananthes sp.; Am spp , Amphora spp, As spp, Asterionella spp.; Ba spp, Bacillaria spp; Ce spp ,Ceratoneis spp; CL, Closteriopsis longissima ; CP, Cocconeis placentula;; Cy spp., Cyclotella spp Cym B, Cymbella bengalensis; Cym c, Cymbella cistuala; De spp, Denticula spp.; Di V, Diatoma vulgaris ; fr spp , Frustulia spp.. FC, fragilaria capucina; Go, Gomphonema spp; Me, Melosira spp; Na, Navicula spp.; NR, Nitzschia recta; Pi spp, Pinnularia spp... SU, Synedra ulna; SA; Synedra acus TF Tabellaria fenestrata ;TFI, Tabellaria flocculosa; AH, Actinastrum hantzschii; AF, Ankistrodesmus falcatus;CG, Cladophora glomerata;Co spp, Cosmarium spp..; Clo spp, Closterium spp..; Mo spp, Mougeotia Spp.. Oo spp, Oocystis spp.. PM, Pandorina morum; Pe spp, Pediastrum spp. SQ, Scenedesmus quadricauda ; Sp spp, Spirogyra spp.. St spp, Staurastrum spp..; UZ, Ulothrix zonata; UT, Ulothrix tenerrima;VA, Volvox aureas; XA, Xanthidium antilopaeum; Zy spp, Zygnema spp. ; An sp , Anabaena sp..; ; Ly sp , Lyngbya sp..; Os sp, Oscillatoria sp..; Sp sp, Spirulina sp..; Ce spp, Ceratium spp.. PC, Peridinium cinctum;As spp. Asterocystis spp.)

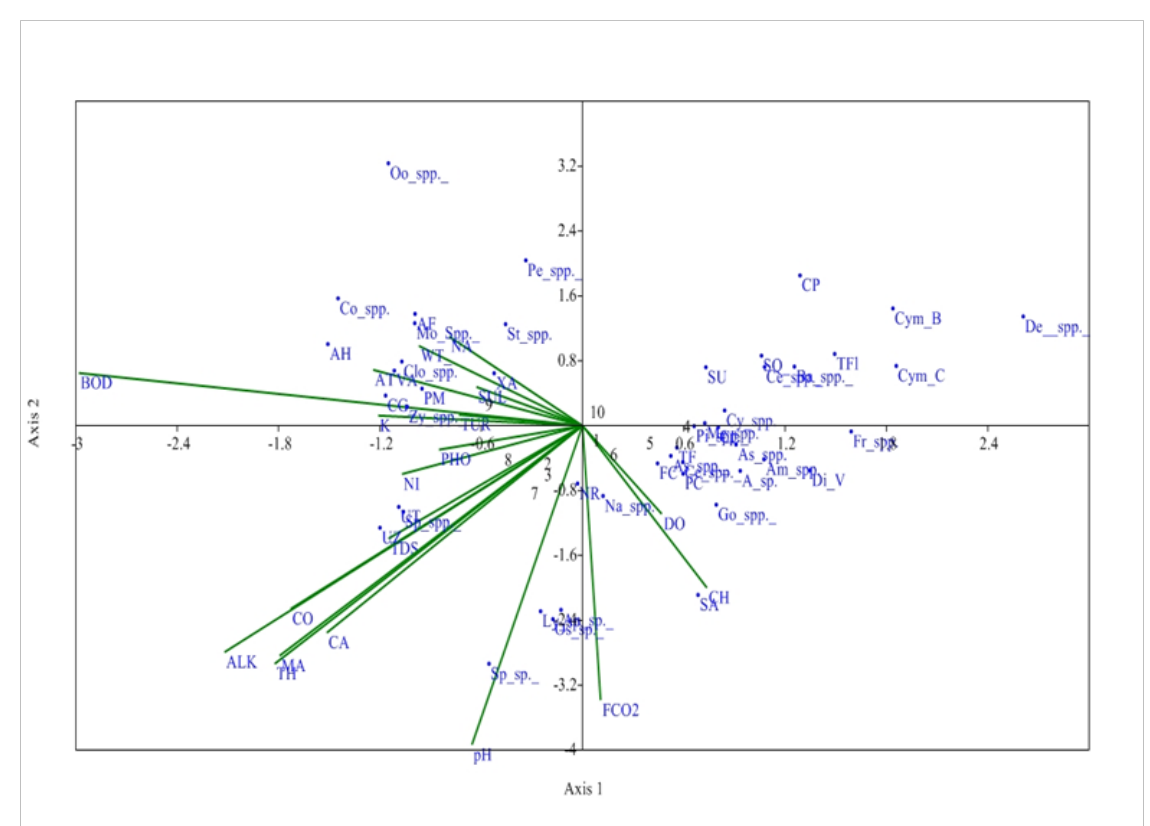

Figure $7 \mathrm{CCA}$ biplot at $\mathrm{S}_{3}$ between physiochemical parameters and phytoplankton species (Physico-chemical parameters, AT, Air temperature; WT, Water temperature ;TUR, Turbidity ; TDS, Total Dissolved Solids; CO, Conductivity ;ALK , Alkalinity; FCO, Free CO; DO, Dissolved Oxygen BOD, Biological Oxygen Demand; pH; CH, Chlorides; TOH, Total hardness; CA, Calcium; MA, Magnesium; NI, Nitrates; PHO, Phosphates; SUL, Sulphates; NA, Sodium ; K, Potassium ); (Phytoplankton species A sp, Achananthes sp.; Am spp , Amphora spp, As spp, Asterionella spp.; Ba spp, Bacillaria spp; Ce spp ,Ceratoneis spp; CL, Closteriopsis longissima ; CP, Cocconeis placentula;; Cy spp., Cyclotella spp Cym B, Cymbella bengalensis; Cym c, Cymbella cistuala; De spp, Denticula spp.; Di V, Diatoma vulgaris ; fr spp , Frustulia spp.. FC, fragilaria capucina; Go, Gomphonema spp; Me, Melosira spp; Na, Navicula spp.; NR, Nitzschia recta; Pi spp, Pinnularia spp.. SU, Synedra ulna; SA; Synedra acus TF Tabellaria fenestrata ;TFI, Tabellaria flocculosa; AH, Actinastrum hantzschii; AF, Ankistrodesmus falcatus;CG, Cladophora glomerata;Co spp, Cosmarium spp..; Clo spp, Closterium spp..; Mo spp, Mougeotia Spp.. Oo spp, Oocystis spp.. PM, Pandorina morum; Pe spp, Pediastrum spp. SQ, Scenedesmus quadricauda ; Sp spp, Spirogyra spp.. St spp, Staurastrum spp..; UZ, Ulothrix zonata; UT, Ulothrix tenerrima;VA, Volvox aureas; XA, Xanthidium antilopaeum; Zy spp, Zygnema spp. ; An sp , Anabaena sp..; ; Ly sp , Lyngbya sp.; Os sp, Oscillatoria sp.; Sp sp, Spirulina sp.; Ce spp, Ceratium spp.. PC, Peridinium cinctum;As spp. Asterocystis spp.) 


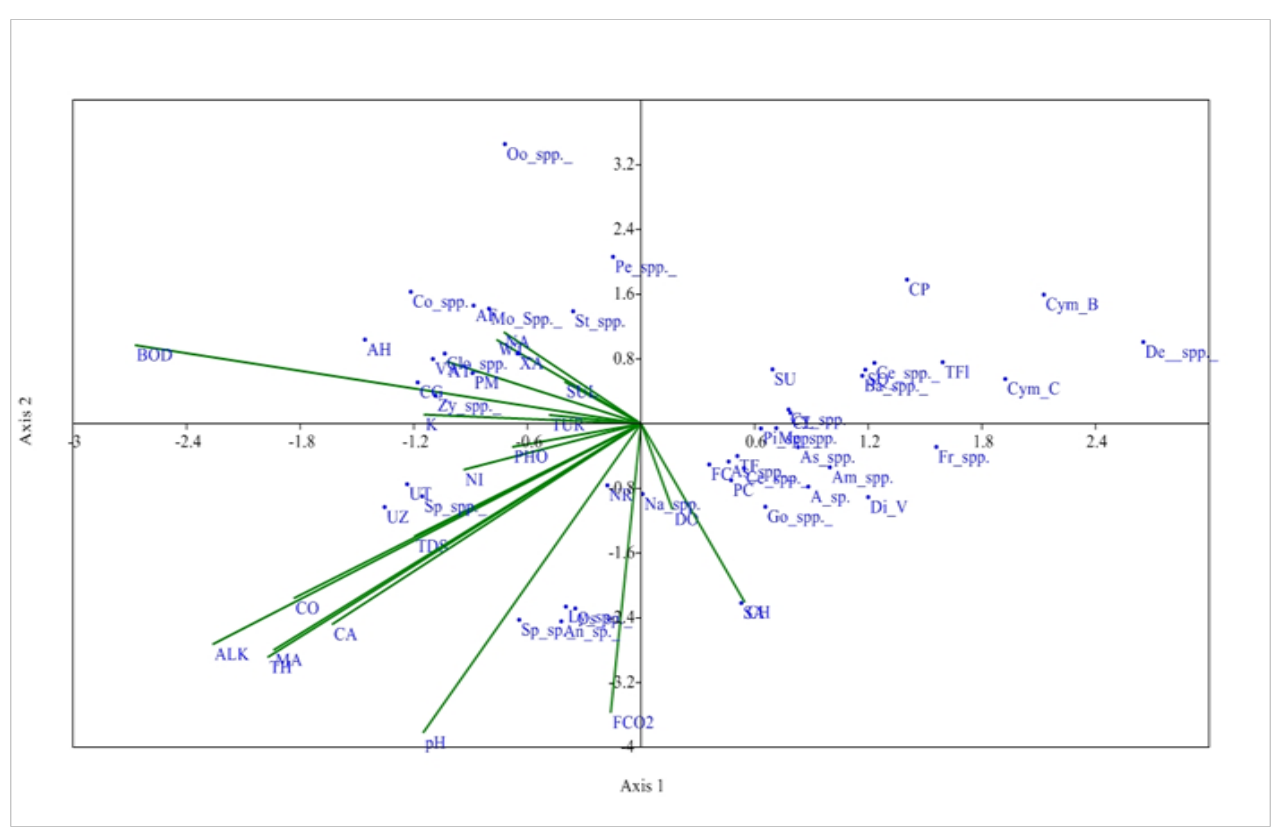

Figure $8 \mathrm{CCA}$ biplot at $\mathrm{S}_{4}$ between physiochemical parameters and phytoplankton species (Physico-chemical parameters, AT, Air temperature; WT, Water temperature ;TUR, Turbidity ; TDS, Total Dissolved Solids; CO, Conductivity; ALK, Alkalinity; FCO, Free $\mathrm{CO}_{2}$; DO, Dissolved Oxygen BOD, Biological Oxygen Demand ; $\mathrm{PH}$,; CH, Chlorides; TOH, Total hardness ; CA, Calcium ; MA, Magnesium; NI, Nitrates; PHO, Phosphates; SUL, Sulphates; NA, Sodium ; K, Potassium ); (Phytoplankton species A sp , Achananthes sp.; Am spp , Amphora spp, As spp, Asterionella spp.; Ba spp, Bacillaria spp; Ce spp ,Ceratoneis spp; CL, Closteriopsis longissima ; CP, Cocconeis placentula;; Cy spp., Cyclotella spp Cym B, Cymbella bengalensis; Cym c, Cymbella cistuala; De spp, Denticula spp.; Di V, Diatoma vulgaris ; fr spp , Frustulia spp.. FC, fragilaria capucina; Go, Gomphonema spp; Me, Melosira spp; Na, Navicula spp.; NR, Nitzschia recta; Pi spp, Pinnularia spp.. SU, Synedra ulna; SA; Synedra acus TF Tabellaria fenestrata ;TFI, Tabellaria flocculosa; AH, Actinastrum hantzschii; AF, Ankistrodesmus falcatus;CG, Cladophora glomerata;Co spp, Cosmarium spp..; Clo spp, Closterium spp..; ; Mo spp, Mougeotia Spp.. Oo spp, Oocystis spp.. PM, Pandorina morum; Pe spp, Pediastrum spp. SQ, Scenedesmus quadricauda ; Sp spp, Spirogyra spp.. St spp, Staurastrum spp..; UZ, Ulothrix zonata; UT, Ulothrix tenerrima;VA, Volvox aureas; XA, Xanthidium antilopaeum; Zy spp, Zygnema spp. ; An sp , Anabaena sp..; : Ly sp, Lyngbya sp..; Os sp, Oscillatoria sp..; Sp sp, Spirulina sp..; Ce spp, Ceratium spp.. PC, Peridinium cinctum; As spp. Asterocystis spp.)

\section{Conclusion}

A marked seasonal fluctuation in density of phytoplankton community along with the physico-chemical variables of Dodi Tal was observed. Dissolved oxygen, chlorides, alkalinity conductivity, total hardness, $\mathrm{pH}$, free $\mathrm{CO}_{2}$, TDS and Magnesium, Calcium affect the distribution of phytoplankton in Dodi Tal. These parameters have significant correlation with the density of phytoplankton. Bacillariophyceae was found to be the dominant group in high altitude wetland, Dodi Tal indicating a considerably healthy condition of the lacustrine ecosystem. However, density of Cyanophyceae and Dinophyceae at $\mathrm{S}_{3}$ and $\mathrm{S}_{4}$ indicated some pollution load at these sites. A high value of Shannon Wiener index is also an indication of good quality of water and health of the high altitude wetland, Dodi Tal, Garhwal Himalaya. The present baseline data on water quality and density of phytoplankton can be a reference for further study and management of the high altitude wetland of Garhwal Himalaya for sustainable delivery of goods and service to large number of trekkers, hikers, tourists and shepherds..$^{29-31}$

\section{Acknowledgements}

One of the authors (Sushma Singh) is thankful to the University Grant Commission and H.N.B Garhwal University (A Central University) for providing Central University fellowship for undertaking the present work.

\section{Conflict of interest}

There is no conflict of interest to declare regarding the publication of this paper.

\section{References}

1. Tian $\mathrm{C}, \mathrm{Pei} \mathrm{H}, \mathrm{Hu}$ W, et al. Variation of cyanobacteria with different environmental conditions in Nansi Lake, China. $J$ Environ. Sci. 2012;24(8):1394-1402.

2. Fott J, Blazo M, Stuchlik E, et al. Phytoplankton in three Tatra mountain lakes of different acidification status. J Limnol. 1999;58(2):107-116.

3. Tolotti M. Phytoplankton and littoral epilithic diatoms in high mountain lakes o f Adamello-Brenta Regional Park (Trentino, Italy) and their relation to trophic status and acidification risk. Journal of Limnology. 2001;60(2):171-188.

4. Ganai AH, Parveen S, Khan AA et al. Phytoplankton diversity at Watlab Ghat in Wular lake, Kashmir. Jour Eco Nat Environ. 2010;2(8):140-146.

5. Bhat FA, Yousuf AR, Aftab A, et al. Ecology and biodiversity in Pangong Tso (lake) and its inlet stream in Ladakh, India. International Journal of Biodiversity and Conservation. 2011;3(10):501-511.

6. Laskar HS, Gupta S. Phytoplankton community and limnology of Chatla floodplain wetland of Barak Valley, Assam, North-East India. Knowl Manag Aquat Ecosyst. 2013;411:6.

7. Jindal R, Thakur RK, Singh UB, et al. Phytoplantons dynamics and water quality of Prashar Lake, Himachal Pradesh. Sustainability of Water Quality and Ecology. 2014;3-4:101-113.

8. Jindal R, Thakur RK, Singh UB, et al. Phytoplankton dynamics and species diversity in a shallow eutrophic, natural mid-altitude lake in Himachal Pradesh (India): role of physicochemical factors. Chemistry and Ecology. 2014,30(4):328-338.

9. Singh VB, Ramanathan AL, Mandal A. Hydrogeochemistry of highaltitude lake: a case study of the Chandra Tal, Western Himalaya, India. 
Arabian Journal of Geosciences. 2016;9:308.

10. Singh Y, Khattar JIS, Singh DP, et al. Limnology and cyanobacteria diversity of high altitude lakes of Lahaul-Spiti in Himachal Pradesh, India. J Biosci. 2014;39(4):643-657.

11. Negi RK, Rajput V. Assessment of phytoplankton diversity in relation to abiotic factors of Nainital Lake of Kumaon Himalyas of Uttarakhand , India. Asian Journal of Scientific Research. 2015;8(2):157-164.

12. Gupta R, Gupta PK. The effect of artificial de-stratification on the water quality of a freshwater high altitude lake. J Ecophysiol Occup Hlth. 2012;12:27-41.

13. Sharma RC, Tiwari V. Phytoplankton diversity in relation to physicochemical environmental variables of Nachiketa Tal, Garhwal Himalaya. Biodiversity International Journal. 2018;2(2):128-136.

14. APHA. Standard Methods for the Examination of Water and Wastewater 21 st ed. American Public Health Association, APHA, AWWA, WEF, Washington; 2005. $1170 \mathrm{p}$.

15. Munshi JD, Roy SP, Munshi JS. Manual of Freshwater Biota. Delhi, India: Narendra Publishing House; 2010:1-435.

16. Bellinger EG, Sigee DC. Freshwater Algae (Identification and Use as Bioindicators). Wiley-Blackwell; 2010:1-243.

17. Yaqoob KU, Pandit AK, Wani SA. Comparative physicochemical limnology of three lakes of Kashmir Himalaya. Proceedings of Taal 2007; The 12th World Lake Conference: 2008:1922-1927.

18. Kumar P, Wanganeo A, Sonaullah F, et al. Limnological study on two high altitude Himalayan ponds, Badrinath, Uttarakhand. Int $J$ Ecosyst. 2012;2(5):103-111.

19. Lami A, Tartari GA, Musazzi S, et al. "High Altitude Lakes: Limnology and Paleolimnology," Mountains Witnesses of Global Changes. Amsterdam: Elsevier; 2007:155-170.

20. Rawat MS, Sharma RC. Phytoplankton population of Garhwal Himalayan lake Deoria Tal, Uttranchal. J Ecophysiol Occup Hith 2005;5:73-76.

21. Naik A, Wanganeo A, IshaqA, et al. Summer limnology of a high mountain
Lake 'Kailash Lake' Bhaderwah, Jammu and Kashmir. International Journal of Environmental Sciences. 2012;3:3.

22. Jindal R, Thakur RK. Diurnal variations of plankton diversity and physicochemical characteristics of Rewalsar Wetland, Himachal Pradesh, India. Recent Research in Science and Technology. 2013;5(3):4-9.

23. Ganai AH, Parveen S. Effect of physico-chemical conditions on the structure and composition of the phytoplankton community in Wular Lake at Lankrishipora, Kashmir. International Journal of Biodiversity and Conservation. 2014;6(1):71-84.

24. Verma PU, Chandawat DK, Solanki HA. Seasonal variation in physicochemical and phytoplankton analysis of Kankaria lake, Ahembabad. Life Sci Leaflets. 2011;19:842-854.

25. Ansari MF, Ankalgi RF, Ankalgi SR. Studies on physico-chemical aspects and plankton of Unkal Lake at Hubli (Karnataka, India) 2008: proceeding of Taal 2007: the 12th World Lake Conference; 2008:1687-1694.

26. Maheshwari K, Vashistha, Paulose PV, et al. Seasonal chnges in Phytoplankton Community of Lake Ramgarh, India. International Journal of Current Microbiolgy and Applied Science. 2015;4(11):318-330.

27. Shekhar STR, Kiran BB, Puttaiah T, et al. Phytoplankton as index of water quality with reference to industrial pollution. $J$ Environ Biol. 2008;29(2):233-236.

28. Thakur RK, Jindal R, Singh UB, et al. Plankton diversity and water quality assessment of three freshwater lakes of Mandi (Himachal Pradesh, India) with special reference to planktonic indicators. Environ Monit Assess. 2013;185(10):1-22.

29. Bhosale LJ, Dhumal SN, Sabale AB. Seasonal variations in occurrence of Phytoplankton and primary productivity of some selected lakes in Maharashtra. The Bioscan. 2010;2:569-578.

30. Brettum P, Andersen T. The use of phytoplankton as indicators of water quality. NIVA report SNO; 2005:4818-2004.

31. Reynolds C, Huszar V, Kruk C, et al. Towards a functional classification of the freshwater phytoplankton. J Plankton Res. 2002;24:417-428. 